\title{
Applications of Survival Analysis in Botanical Epidemiology
}

\author{
H. Scherm and P. S. Ojiambo
}

Department of Plant Pathology, University of Georgia, Athens 30602.

Accepted for publication 29 April 2004.

\begin{abstract}
Scherm, H., and Ojiambo, P. S. 2004. Applications of survival analysis in botanical epidemiology. Phytopathology 94:1022-1026.

Data on the occurrence and timing of discrete events such as spore germination, disease onset, or propagule death are recorded commonly in epidemiological studies. When analyzing such "time-to-event" data, survival analysis is superior to conventional statistical techniques because it can accommodate censored observations, i.e., cases in which the event has not occurred by the end of the study. Central to survival analysis are two mathematical functions, the survivor function, which describes the

or more groups using chi-square-based test statistics. The effects of discrete or continuous covariates on survival times can be quantified with two types of models, the accelerated failure time model and the proportional hazards model. When applied to longitudinal data on the timing of defoliation of individual blueberry leaves in the field, analysis with the accelerated failure time model revealed a significantly $(P<0.0001)$ increased defoliation risk due to Septoria leaf spot, caused by Septoria albopunctata. Defoliation occurred earlier for lower leaves than for upper leaves, but this effect was confounded in part with increased disease severity on lower leaves.
\end{abstract} probability that an individual will "survive" (i.e., that the event will not occur) until a given point in time, and the hazard function, which gives the instantaneous risk that the event will occur at that time, given that it has not occurred previously. These functions can be compared among two
Additional keywords: rabbiteye blueberry, Vaccinium ashei, Weibull distribution.
The analysis of the occurrence and timing of events in a population is a common statistical problem in many disciplines. In this context, an "event" is defined as a qualitative change occurring at a particular point in time (1). In the biomedical sciences, the event of interest is most often the time of death of an individual, measured from the time of disease onset, diagnosis, or the time when a particular treatment (e.g., surgery or chemotherapy) was applied. It is in this discipline that the term survival analysis was coined. In the social sciences, where events of interest might include the timing of arrests, divorces, revolutions, etc., the term event history analysis is used. Time-to-event data are also common in engineering, where the focus is most often on analyzing the time until a piece of equipment fails; this statistical methodology is therefore referred to as failure time analysis in this discipline. Interestingly, the latter term is also used commonly in the ecological literature $(7,15)$. Regardless of the nature of the event of interest, we shall use the term survival analysis throughout this paper; accordingly, the dependent variable will be referred to as survival time.

Data on the occurrence and timing of events are collected routinely during field and laboratory studies on the epidemiology of plant diseases. Examples include the time to first appearance of symptoms in a population of plants or fields; the time to germination in a population of fungal spores; the time to apothecium production in a population of sclerotia; the time to death in a population of nematode eggs; or the time to defoliation in a population of diseased leaves. Despite the common occurrence of survival data in plant pathology, survival analysis is used rarely to examine such data sets. With this paper, we hope to generate

Corresponding author: H. Scherm; E-mail address: scherm@uga.edu

Publication no. P-2004-0719-060

(c) 2004 The American Phytopathological Society more interest in this statistical methodology by reviewing important principles and procedures of survival analysis and by illustrating them with specific examples from our research with Septoria leaf spot of blueberry. More advanced topics and mathematical derivations are available in textbooks on applied survival analysis (5,10-12). Pelz and Klein (18) have provided a detailed comparison of three statistical packages (SAS, SPSS, and BMDP) used commonly for survival analysis. A comprehensive account of the survival analysis features available within the Statistical Analysis System (SAS Institute, Cary, NC) has been published by Allison (1).

Principles of survival analysis. There are several questions an investigator might wish to ask in relation to survival data (Table 1). First, it may be of interest to estimate the survival time distribution for a group of individuals. Among other things, this allows one to calculate the risk that the event will occur within a given interval and compute derived quantities such as the median residual lifetime, i.e., the time to occurrence of the event for an individual that has survived (i.e., not experienced the event) until the beginning of the interval. A second objective might be to compare survival time distributions among two or more groups, e.g., individuals subjected to different treatments following the diagnosis of a disease. Finally, one might wish to quantify the effects of one or more independent variables (covariates) on survival times in an effort to develop a model to describe or predict survival times in a population.

Investigators are often tempted to use conventional statistical methodology for the analysis of survival data. For example, logistic regression analysis could be applied to quantify the importance of certain covariates in classifying individuals into two groups, those that did or did not experience the event during the period of observation. However, this approach can result in a considerable loss of information because differences in the timing of event occurrence are not considered. Alternatively, one could use 
ordinary least-squares regression analysis to identify covariates that influence survival times. The major drawback here lies in the fact that survival data are often censored, i.e., they contain observations for which one does not know when the event occurred, either because the corresponding individual was lost from the data set during the study period, or because the study ended before all individuals experienced the event. Both causes of censoring occur commonly during epidemiological investigations. With conventional statistical methodology, censored observations would either have to be deleted, or one would have to make certain adhoc assumptions, e.g., by setting their survival times to the total time period from onset to termination of the study. By contrast, the likelihood-based parameter estimation methods used in survival analysis can effectively extract relevant information from both censored and uncensored observations, thereby producing reliable parameter estimates $(1,12)$. Furthermore, survival analysis is the only method that can readily accommodate time-dependent covariates, i.e., independent variables whose values change during the course of the study. As an example, consider an epidemiological investigation in which the event of interest is the time to defoliation in relation to leaf disease severity. Disease severity is a time-dependent covariate, given that severity values change over time and they do so differently for each leaf.

Previous uses of survival analysis in botanical epidemiology. With a few exceptions, application of survival analysis in plant pathology has been limited. Neher et al. (16) used the technique to determine survivorship of even- and mixed-aged populations of the two soybean species, Glycine max and G. soja, in relation to pre- and postemergence damping-off caused by Pythium aphanidermatum. The analysis revealed significant effects of plant species as well as a significant species-age structure interaction: disease-induced mortality was greater for G. max than for G. soja, in mixed-aged compared with even-aged populations of G. max, and in even-aged relative to mixed-aged populations of $G$. soja. These effects could be explained based on differences between the two plant species in the duration of the period of seedling susceptibility (a function of age structure) as well as other host and environmental factors that determine seed or seedling susceptibility to infection.

Madden and Nault (14) applied survival analysis to quantify the effects of Spiroplasma kunkelii (the causal agent of corn stunt disease) and Maize bushy dwarf phytoplasma on adult survival of seven species of leafhopper vectors. There were differential effects on vector survival as expressed by the quartiles of the estimated survival time distributions for the various pathogen-vector combinations. For example, median survival time was reduced for five vector species by $S$. kunkelii and for two species by Maize bushy dwarf phytoplasma. Both pathogens tended to have less detrimental effects on vector species that are well adapted to maize and the environment in which maize grows naturally, supporting the hypothesis that a prolonged association between vector and pathogen will result in the selection of highly compatible vector biotypes and pathogen strains.

In an effort to optimize the timing of preharvest inspections for bacterial ring rot (caused by Clavibacter michiganensis subsp. sepedonicus) in seed potato production fields, Westra et al. (21) developed survival analysis models to predict the time from planting to (i) the onset of foliar symptoms of the disease and (ii) the occurrence of maximum incidence of foliar symptoms in the field. The analysis was based on data collected from three cultivars in diverse potato growing regions of the United States during a 3-year period. Depending on location and cultivar, the models predicted 50 to 72 and 83 to 115 days after planting for survival probabilities, $P=0.95$ and 0.05 , for symptom onset and maximum disease incidence, respectively. In other words, at 50 to 72 days after planting, $95 \%$ of the plots had "survived" without showing foliar symptoms of the disease, whereas by 83 to 115 days after planting, only $5 \%$ of the plots had not yet attained their maximum disease incidence. Because the survival models provide estimates of the probability of symptom onset and maximum disease incidence occurring at given times during the growing season, they allow for the prediction of these two events and optimal scheduling of inspections of potato seed production fields for bacterial ring rot.

In one of the first studies to determine the spread of a nonindigenous plant pathogen over a variable landscape, Jules et al. (9) applied survival analysis to determine the extent to which landscape and host-related covariates affected the probability of invasion by Phytophthora lateralis, a fatal root pathogen of Port Orford cedar in the mixed-conifer forests of northern California and southwestern Oregon. In these forests, cedars are clustered along creeks where their roots are in frequent contact with water (8). Analysis of disease spread over a 23-year period showed that inoculum dispersal occurred primarily by vehicles along logging roads; thus, cedars along creeks crossed by roads were more likely to be killed than those along creeks without road crossings. Survival analysis revealed that the time to infection of individual creek populations of cedar (a measure of invasibility of these sites) was associated positively with host abundance, catchment area (a measure of stream flow along the creek), and proximity between a bridge crossing and the nearest cedar. This study demonstrated that increased connectivity among invasible sites by the presence of roads can increase invasion success of a nonindigenous plant pathogen.

Survival analysis applied to Septoria leaf spot of blueberry. Septoria leaf spot, caused by the fungus Septoria albopunctata, can be damaging on certain blueberry cultivars grown in Georgia (20) and other southeastern states (3). The disease is most severe after harvest during late summer and fall when no fruit are present. Leaf infection during this period reduces photosynthesis (19) and can lead to premature defoliation $(2,3,17)$, both of which may have a negative impact on flower bud initiation in the fall and fruit yield in the following spring. Indeed, when blueberry plants were defoliated artificially (by clipping off leaves) in the fall, subsequent flower bud set and yield were reduced $(13,22)$. Based on these considerations, we initiated field experiments to quantify the relationships between leaf spot severity, defoliation, and yield in southern blueberries (20). Here, we use a subset of the data collected during this study to demonstrate the application of various survival analysis procedures. The focus will be on quantifying the effects of host- and disease-related covariates on time to defoliation of individual leaves.

TABLE 1. General applications of survival analysis and their implementation using procedures of the Statistical Analysis System (SAS) ${ }^{\mathrm{a}}$

\begin{tabular}{llc}
\hline Application & Main output & SAS implementation \\
\hline Estimation of survival time distributions & $\begin{array}{c}\text { Survivor function, } S(t) \text {; hazard function, } h(t) \text {; and derived } \\
\text { quantities (e.g., median residual lifetime) }\end{array}$ & PROC LIFETEST \\
$\begin{array}{l}\text { Comparison of survival time distributions } \\
\text { among groups }\end{array}$ & $\begin{array}{c}\text { Test statistics for hypothesis of equality among groups: log- } \\
\text { rank, Wilcoxon, and likelihood ratio tests }\end{array}$ & PROC LIFETEST \\
$\begin{array}{l}\text { Modeling the effect of discrete and/or } \\
\text { continuous covariates on survival times }\end{array}$ & $\begin{array}{c}\text { Parameter estimates and associated significance levels; } \\
\text { hazard ratio }\end{array}$ & PROC LIFEREG \\
\hline $\begin{array}{l}\text { a Similar procedures are available in other statistical packages, e.g., SPSS and BMDP. A detailed comparison of the survival analysis features of the three } \\
\text { packages is given by Pelz and Klein (18). }\end{array}$
\end{tabular}


Field site and data collection. The study was done in an experimental planting of mature 'Premier' rabbiteye blueberry (Vaccinium ashei) at the University of Georgia Horticulture Farm near Athens. The plants remained untreated with fungicides through the entire season. On 28 September 2001, 50 shoots with varying leaf spot severity levels were selected and tagged, and each leaf present on the distal $20 \mathrm{~cm}$ of these shoots was assigned a unique number for tracking over time. The total population of leaves monitored consisted of 567 individuals. A distinction was made between leaves on the upper half of the shoot segment and those on the lower portion of the shoot, with values of 0 and 1 assigned to lower and upper leaves, respectively. At 6- to 12-day intervals through the remainder of the fall, leaves were assessed individually for disease severity (expressed as number of spots per leaf) and time of defoliation (expressed in days after 28 September). The last disease assessment was made on 2 November, after which leaf spot counts became impossible due to natural leaf senescence. Assessment for defoliation continued for another 2 weeks.

The time course of defoliation and disease progression is depicted in Figure 1. Defoliation advanced slowly during the first month of the study followed by a more rapid rate of leaf loss. At the end of the study, $33.9 \%$ of the leaves were still present (censored observations). Average disease severity doubled from the first to the third assessment followed by a decrease toward the last assessment date due to defoliation of the leaves that were affected most severely.

Because the data set contained multiple censored observations (i.e., leaves that had not defoliated by the end of the study) and because time to defoliation, the dependent variable, can be interpreted as a "survival time" (6), we used survival analysis techniques to describe and model the data.

A fundamental assumption of survival analysis is that observations for different individuals are statistically independent. This was a potential concern in the present study where times to defoliation of leaves located on the same shoot could have been correlated. To test for the validity of the independence assumption, we used the ARIMA procedure in SAS release 8.02 to determine whether times to defoliation of leaves within shoots were autocorrelated. Only 3 of the 50 shoots showed significant $(P<$ 0.05 ) autocorrelation patterns (data not shown), suggesting that observations from different leaves on the same shoot were independent.

Survival and hazard functions. As a first step in survival analysis, it is often useful to estimate the survival time distributions of the event of interest. This allows one to calculate derived

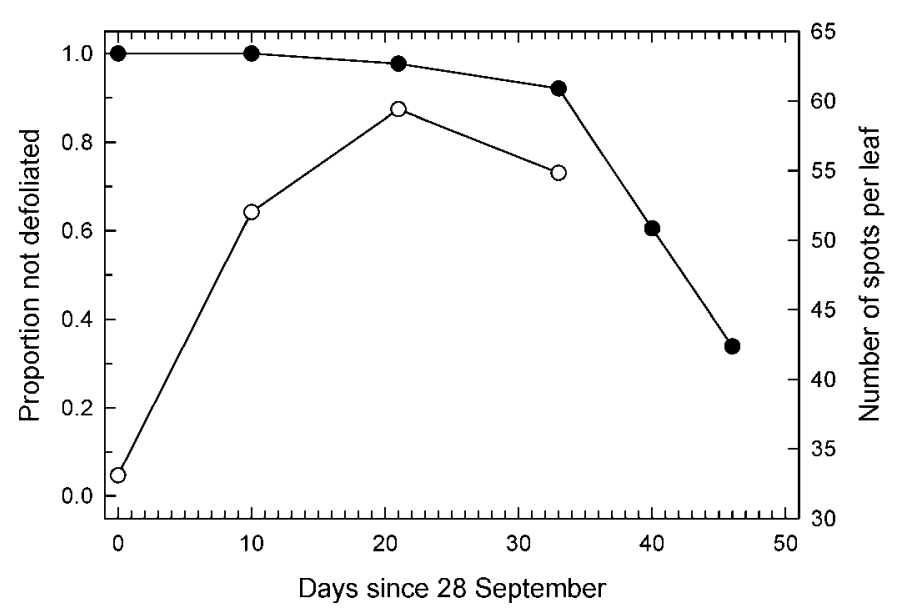

Fig. 1. Temporal progress of Septoria leaf spot severity (O) and defoliation (•) on individual leaves of 'Premier' rabbiteye blueberry in a field study in Georgia in the fall of 2001 . quantities (such as the median residual lifetime) and to compare survival time distributions across treatment groups.

Central to survival analysis are two mathematical functions, the survivor function, $S(t)$, and the hazard function, $h(t) . S(t)$ is a type of cumulative distribution function describing the probability that an individual present at time 0 will survive until time $t$. It is thus defined as $S(t)=\operatorname{Pr}(T \geq t)$, where $T$ is the time of the event (e.g., death) for the individual of interest. By contrast, $h(t)$ is the conditional probability density function describing the instantaneous risk that the event will occur at time $t$, given that it has not occurred previously:

$$
h(t)=\lim _{\Delta t \rightarrow 0} \frac{\operatorname{Pr}(t \leq T<t+\Delta t \mid T \geq t)}{\Delta t}
$$

The hazard is a dimensionless number that may be interpreted as number of events per interval of time (1). Thus, taking the inverse of the hazard gives the expected time until event occurrence. It is important to note that, unlike $S(t), h(t)$ is not bounded by an upper limit of 1.

Survivor and hazard functions may be estimated using two different methods, both of which are available in major statistical packages (18). Briefly, with the Kaplan-Meier method, the survivor function is recalculated after each individual event, making the approach very exact but cumbersome for large data sets. By contrast, the life-table method performs the necessary calculations for specified time intervals, each of which may contain numerous events. Therefore, this approach is most useful for large data sets or when event times are not measured precisely.

Because assessments in the Septoria leaf spot study were made at intervals containing multiple defoliation events, we used the life-table method (implemented with PROC LIFETEST in SAS) to obtain estimates of survivor and hazard functions (Fig. 2). As expected, the estimated survivor function closely resembled the defoliation curve in Figure 1. The hazard function showed that the instantaneous risk of defoliation remained very low for about 1 month after initiation of the study, after which it increased exponentially (Fig. 2). The median residual lifetime of leaves at the beginning of the study was 46.4 days; this value decreased to 6.5 days for leaves still present at the end of the study.

Comparison among groups. Survivor functions are compared readily among two or more treatment groups using chi-squarebased test statistics, of which the log-rank, Wilcoxon, and likelihood ratio tests are the most common. The relative merits of each of these tests are discussed in detail elsewhere $(1,7)$. Briefly, the likelihood ratio test is based on the assumption that the hazard function is constant in each group, which is often implausible

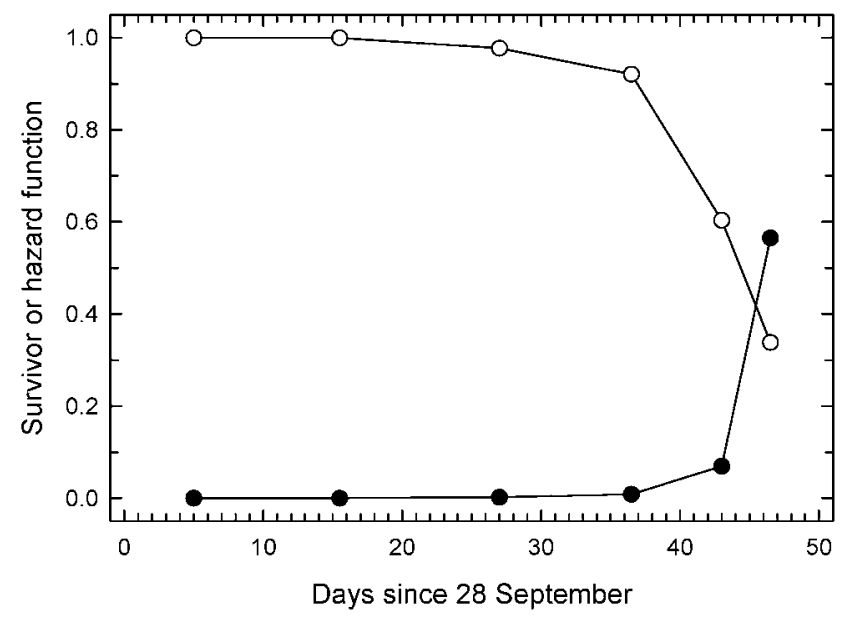

Fig. 2. Life-table estimates of the survivor $(O)$ and hazard $(\bullet)$ functions describing defoliation of individual leaves of 'Premier' rabbiteye blueberry in a field study in Georgia in the fall of 2001. 
with biological data. The log-rank test is most appropriate when proportional hazards assumptions are satisfied (explained below) and when there is limited censoring. Wilcoxon's test is generally superior in all other situations (7).

We applied the LIFETEST procedure to compare the survivor functions of leaves positioned on the upper half of the shoots (i.e., younger leaves; $n=286$ ) and those on the lower sections of the shoots $(n=281)$. The survivor and hazard functions for the two leaf groups were very similar during the first month of the study, after which the defoliation risk of the lower leaves increased relative to that of the upper leaves (Fig. 3A). The difference in survival between the two groups was relatively small but statistically significant $(P<0.0001)$ according to both the log-rank test and the Wilcoxon test. The median residual lifetime of lower and upper leaves at the end of the study was 6.1 and 6.8 days, respectively.

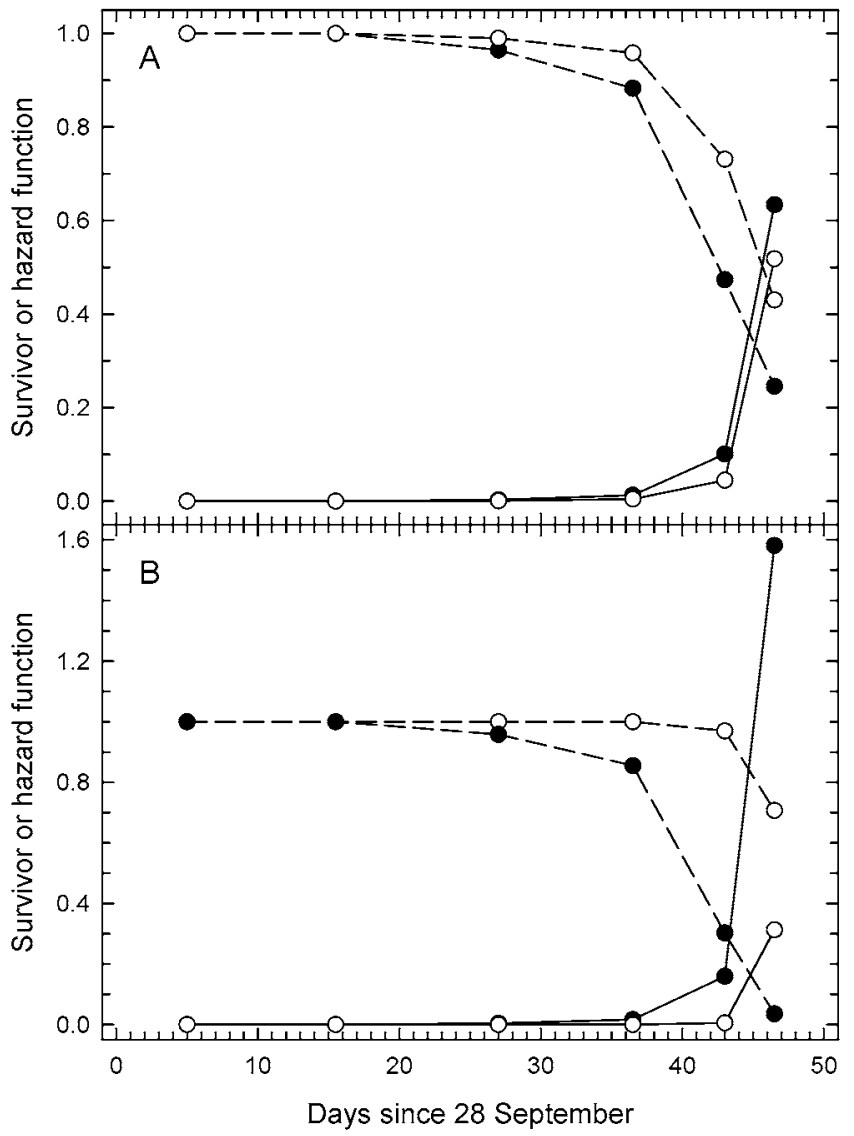

Fig. 3. Life-table estimates of the survivor (- - - -) and hazard (-) functions describing defoliation of individual leaves of 'Premier' rabbiteye blueberry in a field study in Georgia in the fall of 2001. A, Comparison of upper $(\mathrm{O})$ and lower $(\bullet)$ leaves; and $\mathbf{B}$, comparison of leaves with $\leq 10$ Septoria leaf spots $(O)$ and those having $>10$ leaf spots $(\bullet)$ on the first assessment date.
In a second group comparison, we compared defoliation risk for leaves with low disease severity at the beginning of the study (having $\leq 10$ Septoria leaf spots at the first assessment date; $n=$ 256) and those being infected severely at that time ( $>10$ spots at the first assessment; $n=311$ ). The difference in survival between the two groups was pronounced (Fig. 3B) and highly significant $(P<0.0001)$, suggesting that severe infection by $S$. albopunctata is a major contributor to premature defoliation. Indeed, at the end of the study, the defoliation hazard of leaves with high initial leaf spot severity was more than five times greater than that of leaves with low initial disease severity (Fig. 3B).

Modeling survival data. The most powerful approach to survival analysis is based on the use of models to describe and predict the effects of discrete and/or continuous covariates on survival times in a population. Two general modeling approaches are available in most statistical packages, the accelerated failure time (AFT) model and the proportional hazards $(\mathrm{PH})$ model $(1,18)$. The main differences between the two methods are summarized in Table 2. The PH model, also known as Cox regression model (4), is more flexible and easier to use because it does not require the investigator to assume a particular probability distribution representing survival times. This can be advantageous in situations where there are no a-priori biological reasons based on which to choose one distribution over another. The PH approach is based on the premise that, for a given covariate, the hazard functions at different levels of that covariate are proportional. In other words, the hazard functions are similar in shape for different levels of the covariate and only the relative risk of event occurrence at any given point in time is affected. The AFT model is more appropriate in situations where these assumptions are not satisfied, e.g., if the covariate modifies primarily the timing of periods of high hazard (7). In practice, it is relatively straightforward to test whether the $\mathrm{PH}$ assumption is met by developing a PH model that includes an interaction term between time and the covariate of interest, in addition to the covariate itself (12); if the interaction term is statistically significant, the AFT model is more appropriate.

We considered two covariates in developing a model for the timing of defoliation of blueberry leaves, leaf position on the shoot (a binary variable) and disease severity on the last disease assessment date prior to defoliation (a continuous variable). When a PH model was fitted to the data using the PHREG procedure in SAS, the interaction terms between both covariates and time were statistically significant $(P<0.05)$. Thus, the $\mathrm{PH}$ approach was rejected and an AFT model was pursued instead. The model was implemented with PROC LIFEREG in SAS using the Weibull model to describe the underlying survival time distribution. This choice of probability distribution was based on the observation that $h(t)$ increased at an increasing rate over time (Figs. 2 and 3), a property consistent with a Weibull distribution of survival times (1). Defoliation events within two successive assessment dates were treated as interval-censored in the analysis because the exact date of defoliation within the assessment interval was unknown. The resulting AFT model described the data well; the parameter

TABLE 2. General modeling approaches for survival data and their implementation using procedures of the Statistical Analysis System (SAS) ${ }^{\mathrm{a}}$

\begin{tabular}{lll}
\hline Property & Accelerated failure time model & Proportional hazards model \\
\hline Model formulation & $\log _{e} T=\beta_{0}+\beta_{1} X_{1}+\beta_{2} X_{2}$ & $\log _{e} h(t)=\log _{e} \lambda_{0}(t)+\beta_{1} X_{1}+\beta_{2} X_{2}$ \\
Estimation method & Maximum likelihood (parametric) & Partial likelihood (semi-parametric) \\
Underlying survival time distribution & Must be specified (e.g., exponential, Gompertz, Weibull, gamma) & Shape not specified \\
Effect of covariates & Multiplicative effect on survival times & Multiplicative effect on hazard functions \\
Time-dependent covariates & Not available & Readily included \\
SAS implementation & PROC LIFEREG & PROC PHREG \\
\hline
\end{tabular}

${ }^{a}$ Similar procedures are available in other statistical packages, e.g., SPSS and BMDP. A detailed comparison of the survival analysis features of the three packages is given by Pelz and Klein (18).

${ }^{\mathrm{b}}$ Examples are for models with two covariates, $X_{1}$ and $X_{2} . T=$ survival time, $h(t)=$ hazard function, $\lambda_{0}(t)=$ baseline hazard function, and $\beta_{i}=$ parameters to be estimated. The baseline hazard function, which represents the hazard function for an individual whose $X_{i}$ values are all zero, is not estimated. 


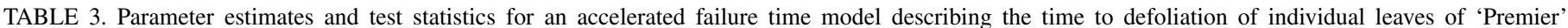
rabbiteye blueberry in a field study in Georgia in the fall of 2001

\begin{tabular}{|c|c|c|c|c|c|}
\hline Parameter $^{\mathrm{a}}$ & df & Estimate & Standard error & Chi-square & $P>$ chi-square \\
\hline Intercept & 1 & 3.88 & 0.0071 & 298,852 & $<0.0001$ \\
\hline Disease severity & 1 & -0.0020 & 0.0001 & $1,372.6$ & $<0.0001$ \\
\hline Leaf position & 1 & 0.0101 & 0.0078 & 1.68 & 0.1946 \\
\hline Weibull scale parameter & 1 & 0.0602 & 0.0034 & $\ldots$ & $\ldots$ \\
\hline Weibull shape parameter & 1 & 16.6 & 0.936 & $\ldots$ & $\ldots$ \\
\hline
\end{tabular}

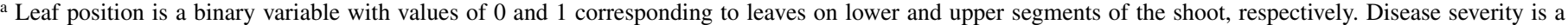
continuous variable, expressed as the number of leaf spots due to Septoria albopunctata on the last disease assessment date prior to defoliation.

estimates and associated test statistics (Table 3 ) revealed that the effect of disease on defoliation risk was highly significant $(P<$ $0.0001)$, while that of leaf position was not $(P=0.1946)$. The latter result conflicts with that obtained by direct comparison of the survivor functions of leaves positioned on lower and upper sections of the shoots. The most likely explanation is that disease severity and leaf position were confounded, i.e., the disease progressed more rapidly and/or attained greater levels on lower (older) leaves. Thus, leaf position was no longer significant when considered jointly with disease severity.

The AFT parameter estimate for disease severity was $\beta=$ -0.0020 (Table 3 ). Recalling that the dependent variable in the AFT model is $\log _{e} T$ (Table 2), this value can be used to compute the percent change in time to defoliation for each additional leaf spot based on the formula $100 \% \times\left(e^{\beta}-1\right)(1)$; based on this calculation, each additional spot during the assessment period decreased the time to defoliation by $0.2 \%$. Similarly, the percent change in defoliation hazard for each one-unit increase in leaf spot severity can be computed as $100 \% \times\left(e^{-\beta / \sigma}-1\right)$, where $\sigma$ is the scale parameter of the Weibull function (Table 3); this conversion indicates that each additional leaf spot increases the defoliation hazard by $3.3 \%$. Because leaf spots caused by $S$. albopunctata can reach substantial numbers on leaves of susceptible rabbiteye blueberry cultivars (the maximum in the present study was 317 spots per leaf, and $>25 \%$ of the leaves had 100 or more spots), the analysis revealed a considerable and highly significant quantitative effect of disease on the risk of defoliation.

\section{SUMMARY AND CONCLUSIONS}

Survival analysis is a powerful technique for analyzing data sets in which the time until an event occurs is the dependent variable and which contain censored observations. The approach consists of estimating survivor and hazard functions, which describe, respectively, the probability that an individual will survive until a given point in time and the instantaneous risk that the event will occur at that time, given that it has not occurred previously. Survivor functions can be compared among two or more groups using chi-square-based test statistics. The effects of discrete and/or continuous covariates on survival times can be quantified with AFT or PH models. When applied to data on the timing of defoliation of individual blueberry leaves, survival analysis revealed a considerable and significant increase in the risk of defoliation due to Septoria leaf spot. Defoliation on lower portions of the shoot occurred earlier than on upper portions of the shoot, but this effect was confounded at least in part with increased disease severity on lower leaves.

\section{ACKNOWLEDGMENTS}

Our research on the epidemiology of Septoria leaf spot of blueberry is funded in part by MBG Marketing, the Southern Region Small Fruit Consortium, and the USDA-CSREES Pest Management Alternatives Program (grant 01-34381-11181). We thank A. Savelle for assistance in data collection.

\section{LITERATURE CITED}

1. Allison, P. D. 1995. Survival Analysis Using the SAS System. A Practical Guide. SAS Institute, Cary, NC.

2. Brannen, P. M., Scherm, H., and Bruorton, M. D. 2003. Fungicidal control of Septoria leaf spot of blueberry, 2002. Fung. Nemat. Tests 58:SMF019.

3. Cline, W. O. 2002. Blueberry bud set and yield following the use of fungicides for leaf spot control in North Carolina. Acta Hortic. 574: 71-74.

4. Cox, D. R. 1972. Regression models and life tables (with discussion). J. R. Stat. Soc. B34:187-220.

5. Cox, D. R., and Oakes, D. 1984. Analysis of Survival Data. Chapman and Hall, London.

6. Dungan, R. J., Duncan, R. P., and Whitehead, D. 2003. Investigating leaf lifespans with interval-censored failure time analysis. New Phytol. 158:593-600.

7. Fox, G. A. 1993. Failure-time analysis: Emergence, flowering, survivorship, and other waiting times. Pages 253-289 in: Design and Analysis of Ecological Experiments. S. H. Scheiner and J. Gurevitch, eds. Chapman and Hall, New York.

8. Hansen, E. M., Goheen, D. J., Jules, E. S., and Ullian, B. 2000. Managing Port Orford cedar and the introduced pathogen Phytophthora lateralis. Plant Dis. 84:4-10.

9. Jules, E. S., Kauffman, M. J., Ritts, W. D., and Carroll, A. L. 2002. Spread of an invasive pathogen over a variable landscape: A nonnative root rot on Port Orford cedar. Ecology 83:3167-3181.

10. Kalbfleisch, J. D., and Prentice, R. L. 2002. The Statistical Analysis of Failure Time Data. 2nd ed. John Wiley \& Sons, Hoboken, NJ.

11. Lawless, J. F. 2003. Statistical Models and Methods for Lifetime Data. 2nd ed. John Wiley \& Sons, Hoboken, NJ.

12. Le, C. T. 1997. Applied Survival Analysis. John Wiley \& Sons, New York.

13. Lyrene, P. M. 1992. Early defoliation reduces flower bud counts on rabbiteye blueberry. HortScience 27:783-785.

14. Madden, L. V., and Nault, L. R. 1983. Differential pathogenicity of corn stunting mollicutes to leafhopper vectors in Dalbulus and Baldulus species. Phytopathology 73:1608-1614.

15. Muenchow, G. 1986. Ecological use of failure time analysis. Ecology 67:246-250.

16. Neher, D. A., Wilkinson, H. T., and Augspurger, C. K. 1992. Progression of damping-off epidemics in Glycine populations of even-age and mixedage structure. Can. J. Bot. 70:1032-1038.

17. Ojiambo, P. S., Scherm, H., and Brannen, P. M. 2002. Septoria leaf spot intensity, defoliation, and yield loss relationships in southern blueberries. (Abstr.) Phytopathology 92(suppl.):S1025.

18. Pelz, C. J., and Klein, J. P. 1996. Analysis of survival data: A comparison of three major statistical packages (SAS, SPSS, BMDP). Tech. Rep. 17, Division of Biostatistics, Medical College of Wisconsin, Milwaukee.

19. Roloff, I., Scherm, H., and van Iersel, M. W. 2004. Photosynthesis of blueberry leaves as affected by Septoria leaf spot and abiotic leaf damage. Plant Dis. 88:397-401.

20. Scherm, H., Brannen, P. M., Ojiambo, P. S., Savelle, A. T., Krewer, G., and Bruorton, M. D. 2003. Blueberry leaf spots: Epidemiology, yield losses, and control. Pages 57-66 in: Proc. Southeast. Blueberry Conf., Savannah, GA.

21. Westra, A. A. G., Arneson, C. P., and Slack, S. A. 1994. Effect of interaction of inoculum dose, cultivar, and geographic location on the development of foliar symptoms of bacterial ring rot of potato. Phytopathology $84: 410-415$

22. Williamson, J. G., and Miller, E. P. 2002. Early and mid-fall defoliation reduces flower bud number and yield of southern highbush blueberry. HortTechnology 12:214-216. 